\title{
Erratum to: Multifactorial discrimination as a fundamental cause of mental health inequities
}

\author{
Mariam Khan ${ }^{1}$, Misja Ilcisin ${ }^{1}$ and Katherine Saxton ${ }^{2 *}$
}

\section{Erratum}

Unfortunately, after publication of this article [1], it was noticed that the tables were not formatted correctly during the production process. The corrected tables can be seen here and the original article has been updated to reflect this.

\author{
Author details \\ ${ }^{1}$ Public Health Program, Santa Clara University, 500 El Camino Real, Santa \\ Clara, CA 95053, USA. ²Department of Biology, Santa Clara University, 500 El \\ Camino Real, Santa Clara, CA 95053, USA.
}

Received: 30 March 2017 Accepted: 31 March 2017

Published online: 15 August 2017

\section{Reference}

1. Khan M, Ilcisin M, Saxton K. Multifactorial discrimination as a fundamental cause of mental health inequities. International Journal for Equity in Health. 2017;16:43. http://doi.org/10.1186/s12939-017-0532-z.

\footnotetext{
* Correspondence: ksaxton@scu.edu

${ }^{2}$ Department of Biology, Santa Clara University, 500 El Camino Real, Santa

Clara, CA 95053, USA
} 
Table 1 Demographics and health characteristics of LGB participants with high and low depression scores

\begin{tabular}{|c|c|c|c|c|c|c|c|}
\hline & \multicolumn{2}{|c|}{ All Participants } & \multicolumn{2}{|c|}{$\begin{array}{l}\text { Low Depression } \\
\text { Scores }\end{array}$} & \multicolumn{2}{|c|}{$\begin{array}{l}\text { High Depression } \\
\text { Scores }\end{array}$} & \multirow[b]{2}{*}{$x^{2}$} \\
\hline & $\mathrm{N}$ & $(\%)$ & $\mathrm{N}$ & $(\%)$ & $\mathrm{N}$ & $(\%)$ & \\
\hline Ethnicity/Race & \multicolumn{2}{|c|}{$\mathbf{N}=395$} & \multicolumn{2}{|c|}{$N=\mathbf{2 4 8}$} & \multicolumn{2}{|c|}{$\mathrm{N}=147$} & \\
\hline White & 134 & 33.92 & 91 & 36.69 & 43 & 29.25 & \multirow{3}{*}{$13.03^{* *}$} \\
\hline Latino/Hispanic & 131 & 33.16 & 66 & 26.61 & 65 & 44.22 & \\
\hline Black/African-American & 130 & 32.91 & 91 & 36.69 & 39 & 26.53 & \\
\hline Sex & \multicolumn{2}{|c|}{$\mathbf{N}=\mathbf{3 9 5}$} & \multicolumn{2}{|c|}{$\mathrm{N}=\mathbf{2 4 8}$} & \multicolumn{2}{|c|}{$\mathrm{N}=147$} & \multirow{3}{*}{$4.07^{*}$} \\
\hline Male & 198 & 50.13 & 134 & 54.03 & 64 & 43.54 & \\
\hline Female & 197 & 49.87 & 114 & 45.97 & 83 & 56.46 & \\
\hline Age & \multicolumn{2}{|c|}{$\mathbf{N}=\mathbf{3 9 5}$} & \multicolumn{2}{|c|}{$\mathbf{N}=\mathbf{2 4 8}$} & \multicolumn{2}{|c|}{$N=147$} & \multirow{9}{*}{7.11} \\
\hline Under 21 years old & 31 & 7.85 & 21 & 8.47 & 10 & 6.8 & \\
\hline 21 to 25 years old & 74 & 18.73 & 48 & 19.35 & 26 & 17.69 & \\
\hline 26 to 30 years old & 90 & 22.78 & 54 & 21.77 & 36 & 24.49 & \\
\hline 31 to 35 years old & 68 & 17.22 & 43 & 17.34 & 25 & 17.01 & \\
\hline 36 to 40 years old & 53 & 13.42 & 33 & 13.31 & 20 & 13.61 & \\
\hline 41 to 45 years old & 41 & 10.38 & 27 & 10.89 & 14 & 9.52 & \\
\hline 46 to 50 years old & 18 & 4.56 & 14 & 5.65 & 4 & 2.72 & \\
\hline Over 50 years old & 20 & 5.06 & 8 & 3.23 & 12 & 8.16 & \\
\hline Nativity & \multicolumn{2}{|c|}{$\mathrm{N}=395$} & \multicolumn{2}{|c|}{$N=248$} & \multicolumn{2}{|c|}{$N=147$} & \multirow{3}{*}{7.11} \\
\hline Born in the U.S. & 327 & 82.78 & 206 & 83.06 & 121 & 82.31 & \\
\hline Born outside the U.S. & 68 & 17.22 & 42 & 16.94 & 26 & 17.69 & \\
\hline Education & \multicolumn{2}{|c|}{$\mathrm{N}=395$} & \multicolumn{2}{|c|}{$N=\mathbf{2 4 8}$} & \multicolumn{2}{|c|}{$N=147$} & \\
\hline Less than High School Diploma & 38 & 9.62 & 17 & 6.85 & 21 & 14.29 & \\
\hline $\begin{array}{l}\text { High School Diploma or } \\
\text { Equivalent }\end{array}$ & 46 & 11.65 & 34 & 13.71 & 12 & 8.16 & \\
\hline $\begin{array}{l}\text { Some College or Associates } \\
\text { Degree }\end{array}$ & 120 & 30.38 & 70 & 28.23 & 50 & 34.01 & $13.79^{*}$ \\
\hline Bachelor's Degree & 119 & 30.13 & 79 & 31.85 & 40 & 27.21 & \\
\hline Any Post-Bachelors Education & 70 & 17.72 & 48 & 19.36 & 22 & 14.96 & \\
\hline Other & 2 & 0.51 & 0 & 0.00 & 2 & 1.36 & \\
\hline Employment & & 395 & & & & & \\
\hline Employed & 233 & 58.99 & 158 & 63.71 & 75 & 51.02 & $6.14^{*}$ \\
\hline Unemployed & 162 & 41.01 & 90 & 36.29 & 72 & 48.98 & \\
\hline Income & & 386 & & & & & \\
\hline Lowest Quintile & 123 & 31.87 & 69 & 28.51 & 54 & 37.50 & \\
\hline Second Quintile & 124 & 32.12 & 73 & 30.17 & 51 & 35.42 & \\
\hline Third Quintile & 86 & 22.28 & 66 & 27.27 & 20 & 13.89 & $10.62^{*}$ \\
\hline Fourth Quintile & 45 & 11.66 & 29 & 11.98 & 16 & 11.11 & \\
\hline Highest Quintile & 8 & 2.07 & 5 & 2.07 & 3 & 2.08 & \\
\hline Specific Health Outcome Diagnoses & & 395 & & & & & \\
\hline Diabetes & 14 & 3.54 & 8 & 3.23 & 6 & 4.08 & 0.20 \\
\hline Asthma & 99 & 25.06 & 62 & 25.00 & 37 & 25.17 & 0.00 \\
\hline HIV/AIDS & 49 & 12.41 & 31 & 12.50 & 18 & 12.24 & 0.00 \\
\hline Emotional Disorder & 159 & 40.25 & 82 & 33.06 & 77 & 52.38 & $14.32^{* *}$ \\
\hline & & 387 & & & & & \\
\hline Substance Use Disorder & 149 & 38.50 & 86 & 35.10 & 63 & 44.37 & 3.26 \\
\hline
\end{tabular}


Table 2 Effect of discrimination on health outcomes

\begin{tabular}{|c|c|c|}
\hline Logistic Regression & High Depression Score & Substance Use Disorder \\
\hline & $\mathrm{N}=395$, adjusted-OR $(95 \% \mathrm{CI})$ & $\mathrm{N}=387$, adjusted-OR $(95 \% \mathrm{CI})$ \\
\hline Constant & $0.04(0.01-0.12)^{* *}$ & $0.10(0.03-0.31)^{* *}$ \\
\hline White & Reference & Reference \\
\hline Latino/Hispanic & $1.87(1.11-3.13)^{*}$ & $0.92(0.55-1.52)$ \\
\hline Black/African American & $0.76(0.44-1.30)$ & $0.52(0.30-0.88)^{*}$ \\
\hline Female & $1.58(1.03-2.43)^{*}$ & $1.16(0.76-1.78)$ \\
\hline Discrimination & $2.23(1.54-3.23)^{* *}$ & $2.12(1.47-3.06)^{* *}$ \\
\hline $\mathrm{R}^{2}$ & 0.07 & 0.04 \\
\hline Linear Regression & \multicolumn{2}{|c|}{ Psychological Well-being } \\
\hline & \multicolumn{2}{|c|}{$\mathrm{N}=396$, adjusted $-\beta(95 \% \mathrm{CI})$} \\
\hline Constant & \multicolumn{2}{|c|}{$6.23(5.86-6.61)^{* *}$} \\
\hline White & \multicolumn{2}{|c|}{ Reference } \\
\hline Latino/Hispanic & \multicolumn{2}{|c|}{$-0.29(-0.47--0.11)^{* *}$} \\
\hline Black/African American & \multicolumn{2}{|c|}{$-0.05(-0.23--0.13)$} \\
\hline Female & \multicolumn{2}{|c|}{$-0.01(-0.16--0.13)$} \\
\hline Discrimination & \multicolumn{2}{|c|}{$-0.30(-0.42--0.18)^{* *}$} \\
\hline $\mathrm{R}^{2}$ & \multicolumn{2}{|c|}{0.08} \\
\hline \multicolumn{3}{|c|}{$* \mathrm{p}<0.05, * * \mathrm{p}<0.01, \mathrm{OR}=$ odds ratio, $\beta=$ coefficient, $\mathrm{CI}=$ confidence interval } \\
\hline
\end{tabular}

Table 3 Effect of discrimination on risk factors for high depression scores

\begin{tabular}{lcc}
\hline Linear Regression & Chronic Strain & Total Stressful Events \\
\hline Constant & $\mathrm{N}=388$, adjusted- $\beta(95 \% \mathrm{CI})$ & $\mathrm{N}=396$, adjusted- $\beta(95 \% \mathrm{CI})$ \\
White & $0.99(0.89-1.10)^{* *}$ & $2.02(-0.62-4.66)$ \\
Latino/Hispanic & Reference & Reference \\
Black/African-American & $0.12(0.07-0.17)^{* *}$ & $1.55(0.29-2.81)^{*}$ \\
Female & $0.07(0.02-0.12)^{* *}$ & $1.53(0.27-2.79)^{* *}$ \\
Discrimination & $0.05(0.01-0.09)^{*}$ & $0.57(-0.45-1.59)$ \\
\hline $\mathrm{R}^{2}$ & $0.13(0.09-0.16)^{* *}$ & $2.62(1.78-3.46)^{* *}$ \\
\hline${ }^{*} \mathrm{p}<0.05,{ }^{* *} \mathrm{p}<0.01, \beta=$ coefficient, $\mathrm{CI}=$ confidence interval & 0.11 \\
\hline
\end{tabular}


Table 4 Effect of discrimination on protective factors for high depression scores $(N=396)$

\begin{tabular}{lcc}
\hline $\begin{array}{l}\text { Linear Regression } \\
\text { Individual Factors }\end{array}$ & Mastery & Self-Esteem \\
\hline \multicolumn{1}{c}{ adjusted- $\beta(95 \% \mathrm{CI})$} & adjusted- $\beta(95 \% \mathrm{CI})$ \\
\hline White & $2.93(2.77-3.09)^{* *}$ & $3.95(3.68-4.22)^{* *}$ \\
Latino/Hispanic & Reference & Reference \\
Black/African-American & $-0.14(-0.22--0.07)^{* *}$ & $-0.04(-0.17-0.09)$ \\
Female & $-0.02(-0.10-0.06)$ & $0.20(0.07-0.32)^{* *}$ \\
Discrimination & $0.01(-0.05-0.07)$ & $-0.03(-0.14-0.07)$ \\
\hline $\mathrm{R}^{2}$ & $-0.11(-0.16--0.06)^{* *}$ & $-0.27(-0.35--0.18)^{* *}$ \\
\hline Linear Regression & 0.08 & 0.11 \\
Interpersonal Factors & Collective Esteem & Size of Social Support Network \\
\hline & adjusted- $\beta(95 \% \mathrm{CI})$ & adjusted- $\beta(95 \% \mathrm{CI})$ \\
\hline Constant & $5.26(4.82-5.70)^{* *}$ & $6.44(4.94-7.94)$ \\
White & Reference & Reference \\
Latino/Hispanic & $-0.42(-0.63--0.21)^{* *}$ & $-2.04(-2.75--1.33)^{* *}$ \\
Black/African-American & $-0.17(-0.38-0.04)$ & $-1.61(-2.32--0.89)^{* *}$ \\
Female & $0.16(-0.02-0.33)$ & $0.46(-0.12-1.04)$ \\
Discrimination & $-0.04(-0.18-0.10)$ & $0.04(-0.44-0.51)$ \\
\hline $\mathrm{R}^{2}$ & 0.03 & 0.08 \\
\hline$* \mathrm{p}<0.05, * * \mathrm{p}<0.01, \beta=$ coefficient, $\mathrm{CI}=$ confidence interval & \\
\hline & & \\
\hline
\end{tabular}


Table 5 Effect of discrimination on depression and mental health outcomes after controlling for related risk factors

\begin{tabular}{|c|c|c|}
\hline LGB Individuals & \multicolumn{2}{|c|}{ Logistic Regression of High Depression Scores } \\
\hline & \multicolumn{2}{|c|}{$\mathrm{N}=387$, adjusted-OR $(95 \% \mathrm{CI})$} \\
\hline Constant & \multicolumn{2}{|c|}{$0.00(0.00-0.01)^{* *}$} \\
\hline White & \multicolumn{2}{|c|}{ Reference } \\
\hline Latino/Hispanic & \multicolumn{2}{|c|}{$1.43(0.82-2.49)$} \\
\hline Black/African-American & \multicolumn{2}{|c|}{$0.59(0.32-1.06)$} \\
\hline Female & \multicolumn{2}{|c|}{$1.44(0.91-2.27)$} \\
\hline Chronic Strain & \multicolumn{2}{|c|}{$21.62(6.80-68.81)^{* *}$} \\
\hline Total Stressful Events & \multicolumn{2}{|c|}{$1.00(0.96-1.04)$} \\
\hline Discrimination & \multicolumn{2}{|c|}{$1.68(1.12-2.53)^{*}$} \\
\hline $\mathrm{R}^{2}$ & \multicolumn{2}{|c|}{0.13} \\
\hline \multirow[t]{2}{*}{$\begin{array}{l}\text { LGB Individuals with } \\
\text { Low Depression Scores }\end{array}$} & $\begin{array}{c}\text { Linear Regression of Aggregate } \\
\text { Mental Health Scores }\end{array}$ & $\begin{array}{c}\text { Logistic Regression of Anxiety } \\
\text { Scores }\end{array}$ \\
\hline & $\mathrm{N}=245$, adjusted $-\beta(95 \% \mathrm{CI})$ & $\mathrm{N}=242$, adjusted-OR $(95 \% \mathrm{CI})$ \\
\hline Constant & $65.00(56.89-73.10)^{* *}$ & $0.08(0.01-0.68)^{*}$ \\
\hline White & Reference & Reference \\
\hline Latino/Hispanic & $2.96(0.23-5.69)^{*}$ & $0.66(0.33-1.32)^{* *}$ \\
\hline Black/African-American & $2.39(-0.13-4.90)$ & $0.39(0.20-0.76)^{* *}$ \\
\hline Female & $-1.15(-3.27-0.96)$ & $0.83(0.48-1.44)$ \\
\hline Chronic Strain & $-5.76(-11.57-0.05)$ & $2.15(0.48-9.62)$ \\
\hline Total Stressful Events & $-0.03(-0.29-0.18)$ & $0.99(0.94-1.05)$ \\
\hline Discrimination & $-2.39(-4.41--0.38)^{*}$ & $2.07(1.20-3.57)^{* *}$ \\
\hline $\mathrm{R}^{2}$ & 0.05 & 0.05 \\
\hline
\end{tabular}

